\title{
Use of Marker Computed Tomography as a Navigational Tool for Performing Minicraniotomies
}

\author{
Rajnish Kumar Arora ${ }^{1}$ jitendra Shakya ${ }^{1}$ Saravanan Sadhasivam ${ }^{1} \quad$ Rajashekar Rekapalli $^{1}$ \\ Radhey Shyam Mittal ${ }^{1}$ \\ ${ }^{1}$ Department of Neurosurgery, All India Institute of Medical Sciences, \\ Rishikesh, Uttarakhand, India \\ Address for correspondence Saravanan Sadhasivam, MCh, \\ Department of Neurosurgery, All India Institute of Medical Sciences, \\ Rishikesh, India (e-mail: drsaravns@gmail.com).
}

Indian J Neurosurg 2023;12:79-82.

\begin{abstract}
Keywords

- marker computerized tomography

- stereotaxic

- localization

We want to highlight a simple technique for performing smaller craniotomies, using a marker computed tomography scan, which does not require any special equipment, training or cost and is not time-consuming. Pictorial description of two such cases have been provided.
\end{abstract}

\section{Introduction}

Minimal invasive approaches are increasingly being utilized in neurosurgery. In this context, localization of small subcortical lesions prior to craniotomy is an important part of surgical plan. This becomes even more important when lesion is located at or near eloquent locations. Missing target by small margin can result in more brain retraction and/or need of enlarging bony window. ${ }^{1}$ Trainees often tend to use larger craniotomies for relatively smaller lesions to avoid such mishaps. In modern-day neurosurgery, various techniques are available for accurate intraoperative localization of intracranial lesions and thus for tailoring craniotomies, for example, use of stereotaxic apparatus, neuronavigation and intraoperative imaging. However, they can be costly and require some training. Their availability is scarce in resource-limited settings and at tier II hospitals.

We want to highlight a simple technique for performing smaller craniotomies, using a marker computed tomography (CT) scan, which does not require any special equipment, training or cost and is not time-consuming. Pictorial description of two such cases have been provided ( - Figs. 1 A-D and - Figs. 2 A-D).

article published online May 25, 2021
DOI https://doi.org/ 10.1055/s-0041-1730874. ISSN 2277-954X.

\section{Discussion}

Physicians, particularly residents/trainees, may experience difficulty in placing an abnormality seen on the CT brain scan into its precise anatomical localization. This is particularly important in uppermost CT sections, where exact anteroposterior localization of the anatomy viewed varies from one patient to another due to the alteration of the angle at which the scan was performed. Thus, a small parasagittal lesion may appear to be anterior or posterior to its actual location, depending upon whether the scan was done at +15 o or $+20^{\circ}$ to Reid's baseline (infraorbital-meatal line). ${ }^{2}$ Consequently, even for smaller lesions, one may end up performing a larger craniotomy to avoid intraoperative surprises. Although MRI is now an easily available technique that offers multiplane reconstruction/views for localization, however, it is not a good tool for visualizing bony landmarks of cranium, which trainees often use for marking the incision and craniotomy. MRI may be not possible in claustrophobic patients or those with pacemakers.

Historically, CT combined with skull X-rays has been used to localize lesions near to vertex of head, even

(c) 2021. Neurological Surgeons' Society of India. All rights reserved. This is an open access article published by Thieme under the terms of the Creative Commons Attribution-NonDerivative-NonCommercial-License, permitting copying and reproduction so long as the original work is given appropriate credit. Contents may not be used for commercial purposes, or adapted, remixed, transformed or built upon. (https://creativecommons.org/ licenses/by-nc-nd/4.0/)

Thieme Medical and Scientific Publishers Pvt. Ltd., A-12, 2nd Floor, Sector 2, Noida-201301 UP, India 

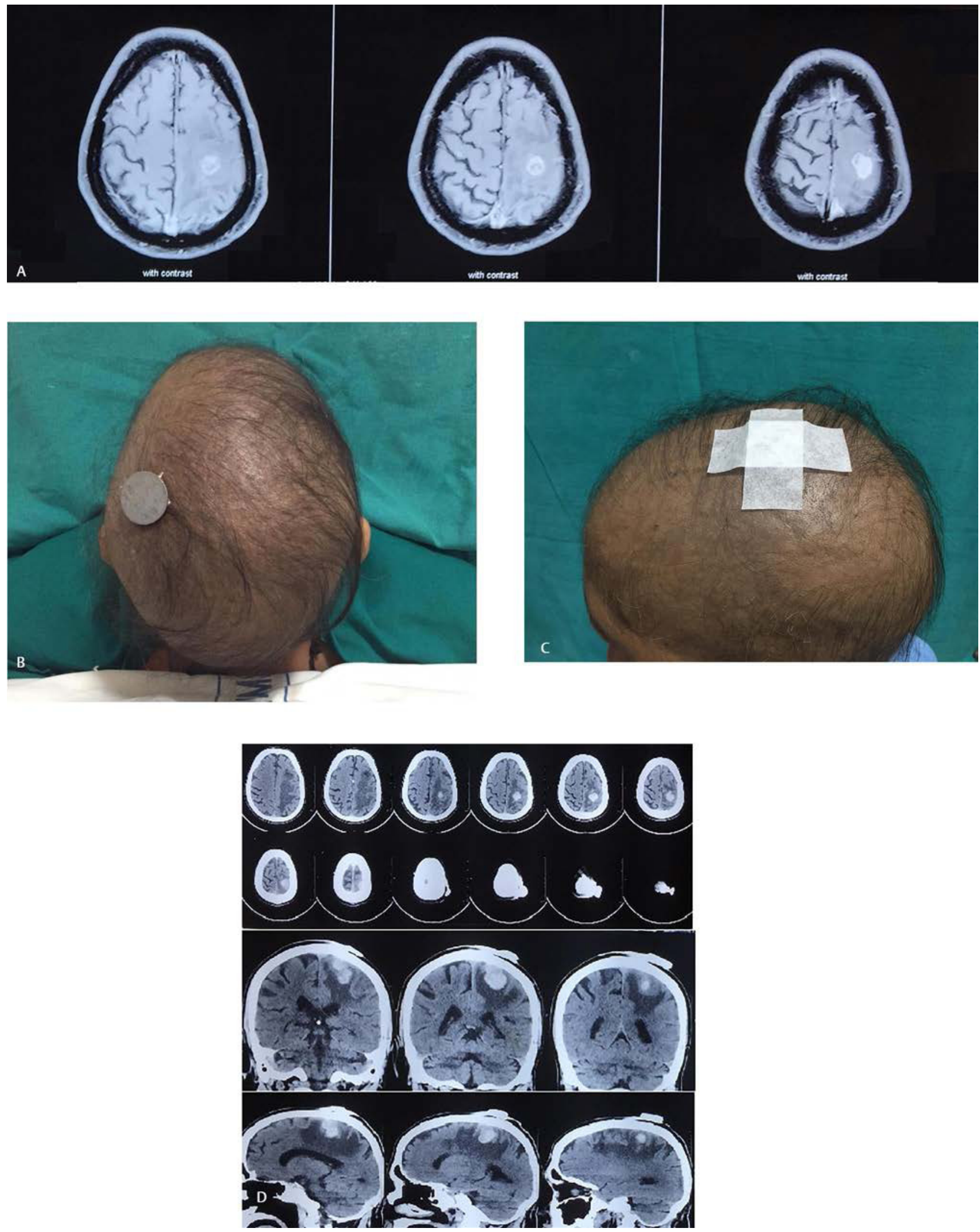

Fig. 1 (A) A 63-year-old male presented with sudden onset and rapidly progressive weakness of right-sided limbs due to a small subcortical lesion deep to motor cortex of left side with hemorrhage. Significant edema and mass effect was present around lesion. (B and C) A marker CT was obtained by placing a metallic coin over site of lesion, as estimated by surface landmarks, secured with help of a tape. Patient was shifted to operating room with coin fixed with tape over scalp. (D) With the help of coronal and sagittal reconstruction images of this marker CT, a linear incision was planned. Complete excision of extra-axial lesion was performed using a small craniotomy. Patient improved well. Histopathological examination (HPE) of lesion was metastasis from renal cell carcinoma. 

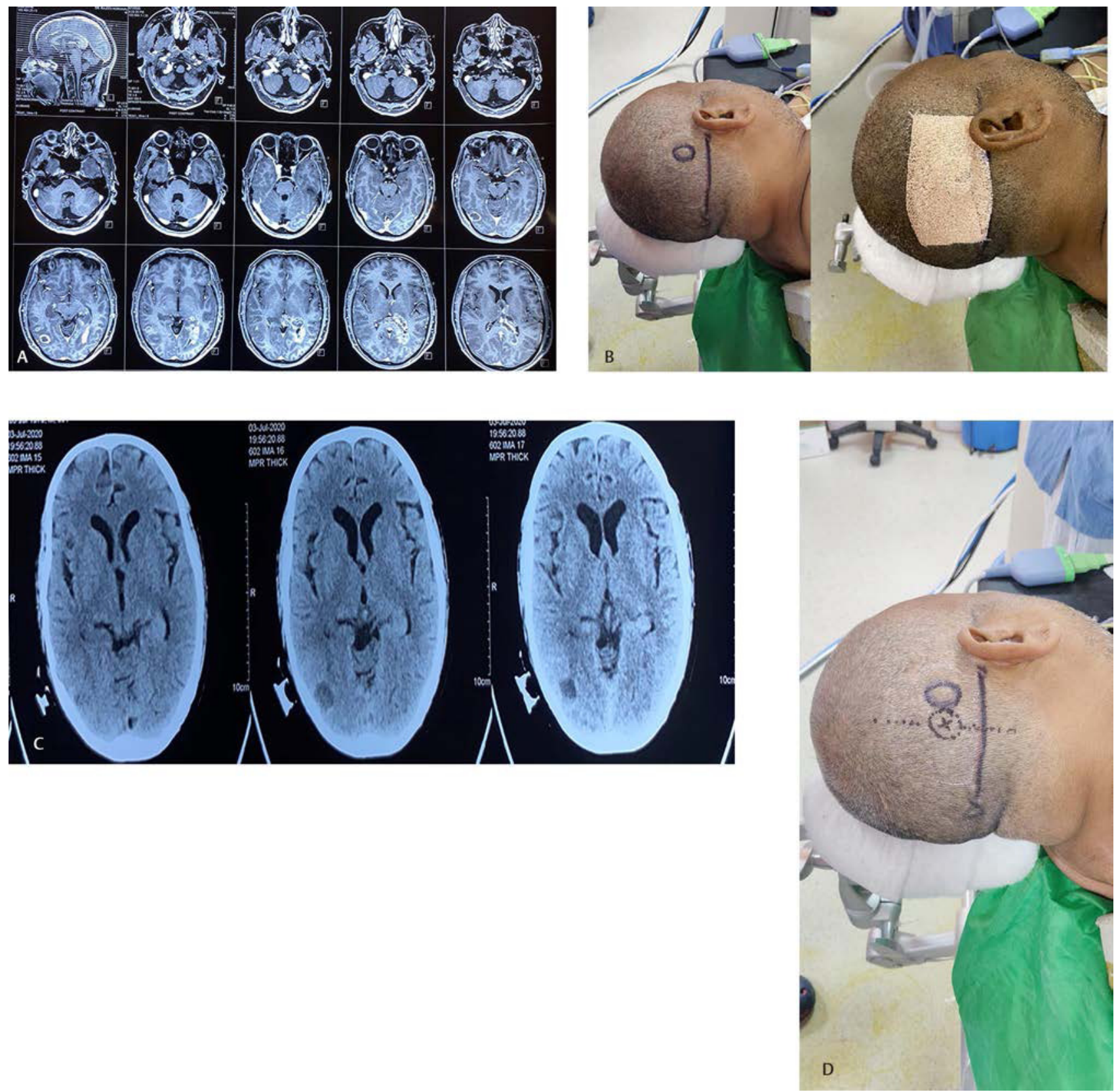

Fig. 2 (A) Axial contrast MRI brain of a 50-year-old male with headache and progressive mental decline demonstrated lesions, affecting body and splenium of corpus callosum with left side intraventricular extension as well as in right occipital lobe. A biopsy from relatively superficial right occipital lesion was planned. (B) A marker was attached on estimated location of right occipital lesion by craniometrics points. (C) Marker CT was obtained by placing a marker on location, estimated by craniometric points. (D) Dotted circle with a cross reveals the precise location of lesion from information provided by marker CT. A linear incision was planned, based on location from marker CT, and lesion was successfully removed by a small craniotomy. Histopathological examination (HPE) of lesion was grade IV glioma.

without use of coronal and sagittal reconstruction. CT has also been used to calculate coordinates for stereotaxic biopsies for long. ${ }^{3-5}$ However, use of multiplane reconstruction, as used in above technique, can obviate use of other adjuncts like X-rays. This type of technique has been employed in the past, particularly for lesions in parasagittal location and those located over high convexity, with tilting or maneuvering the head within gantry or with the use of scalp-mounted fiducial devices like syrinx shape array of radio opaque catheters or plastic grids. ${ }^{1,4,6}$ However, placement of complex fiducial device and interpretation of dots on imaging are critical and may require a learning curve. Further, one either has to fabricate such devices locally or procure it. However, the technique suggested above is simple, as it does not require any device, except a coin (which is usually available, costs only a few pennies/paisa, and reusable) and does not require any training. One can localize lesions near 
convexity easily and plan a smaller craniotomy accordingly without use of any special equipment. Residents or trainees can also verify their craniotomy planning, which was based on anatomical landmarks, thus improving their skills.

\section{Note}

The work pertaining to this study was performed at the All India Institute of Medical Sciences, Rishikesh, India.

\section{Funding}

None.

\section{Conflict of Interest} None declared.

\section{References}

1 Nayak PK, Dutta J. Craniomapper for accurate localization of lesion during craniotomy: how much benefit does it have over anatomical marking? Report of two cases. J Neurosci Rural Pract 2014;5(02):202-203

2 O'Leary DH, Lavyne MH. Localization of vertex lesions seen on CT scan. J Neurosurg 1978;49(01):71-74

3 Gleason CA, Wise BL, Feinstein B. Stereotactic localization (with computerized tomographic scanning), biopsy, and radiofrequency treatment of deep brain lesions. Neurosurgery 1978;2(03):217-222

4 Spincemaille GH, Versteege CW, Blaauw G. Computed tomographyguided scalp marking of cerebral surface lesions; an alternative to stereotaxis for small convexity lesions. Eur J Radiol 1990;11(02):143-144

5 Cail WS, Morris JL. Localization of intracranial lesions from CT scans. Surg Neurol 1979;11(01):35-37

6 Hirschberg H. Localization of brain tumors with a simple scalp-mounted fiducial device. Technical note. J Neurosurg 1989;70(02):280-281 